\title{
DEFECTS LOCATION ESTIMATION USING MULTISCALE THERMAL FINITE ELEMENT METHOD
}

\author{
YVES BRESSON ${ }^{1,2}$, AMÈVI TONGNE ${ }^{1}$, LIONEL ARNAUD ${ }^{1}$ AND MAHER BAILI ${ }^{1}$ \\ ${ }^{1}$ Laboratoire Génie de Production, INP ENIT \\ 47 Avenue d'Azereix, 65000 Tarbes, FRANCE \\ yves.bresson@enit.fr, https://www.lgp.enit.fr/en/index.html \\ ${ }^{2}$ Halbronn \\ Z.I. Lognes, rue des Campanules, 77437 Marne-La-Vallée, FRANCE \\ y.bresson@halbronn.com, http://www.halbronn.com/
}

Key words: Additive Manufacturing, Multiscale approach, Thermal simulation, Selective Laser Melting.

\begin{abstract}
The Selective Laser Melting (SLM) process is the subject of numerous researches for a few decades. Manufactured parts experience very high heating and cooling rates along the laser beam path and suffer an extensive range of temperatures. These high heating and cooling rates can lead to defects such as cracks, distortions, and porosities. These defects are driven by the thermal history, related to several factors such as scanning strategy, surrounding powder insulation, parts geometry, number of parts to be manufactured, recoating time, etc. All these factors need to be considered to precisely simulate the thermal history. In this study, a multiscale thermal approach has been developed and applied to a study case. Most of the thermal history aspects are covered using five temporal and spatial scales FEM model. The overall thermal history and future improvements for simulating a full build plate in reasonable computational time are discussed.
\end{abstract}

\section{INTRODUCTION}

The Selective Laser Melting process is a metal additive manufacturing process widely adopted in the industry and research fields since the last few decades. It consists of scanning a thin (tens of microns thick) metal powder layer using a high-power laser heat source. The process's attractiveness relies on its ability to generate highly complex geometries from materials that could be challenging to manufacture otherwise. This flexibility allows the manufacturing of lightweight, functional industrial parts of elevated added value.

However, there remains some cost and technical challenges to tackle for wider industrial adoption of the process, including the prediction of porosities, distortions, and residual stresses generated from the extremely high heating and cooling rates [1].

The computation of the thermal history requires a strict consideration of the numerous phenomena occurring during the manufacturing process at different scales, i.e. the scanning strategy, the layering delay, the heat accumulation due to the part geometry and complete build plate, etc. These aspects affect the thermal fields that influence regions' heating and cooling 
rates along the laser beam path.

Several methods could have been used to simulate the thermal history of the SLM process, such as adaptative mesh schemes [2], superposition approaches [3], use of GPU hardware to accelerate the computation [4], and multiscale approaches [5].

Multiscale approaches usually consider the thermal aspects at the scanning-track or layer scale only [5]. These thermal outputs are then used in the thermomechanical resolution [6]. However, the previously mentioned thermal aspects could not be fully captured if every scale from macroscale to microscale is not considered. Recent works have shown a correlation between the temperature attained locally and the distribution of porosities within the part [7]. Hence, simulation of thermal history may be directly used to predict location of such defects.

In this study, a novel multiscale approach is presented. It links the thermal outputs of five distinct scales together. Each scale is described and the captured thermal aspects as well as the heat sources' modeling assumptions are listed.

\section{MULTISCALE APPROACH DESCRIPTION AND STUDY CASE}

The multiscale approach comprises five conjugate spatial and temporal levels. Each scale passes the computed thermal data along to the next.

An industrial part, which has been the subject of a previous research paper [8], was chosen as a study case. The part is an hydraulic join used in the aeronautic industry and can be seen Figure 1a, as a topologically optimized shape, machined on functional surfaces. The FEM

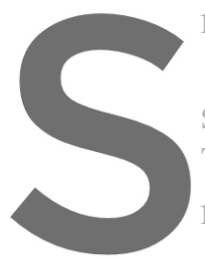
model was realized usin

The main reason fo spatial resolution require Table 1 lists the spatial and temporal domain multiscale approach from macroscale to micro
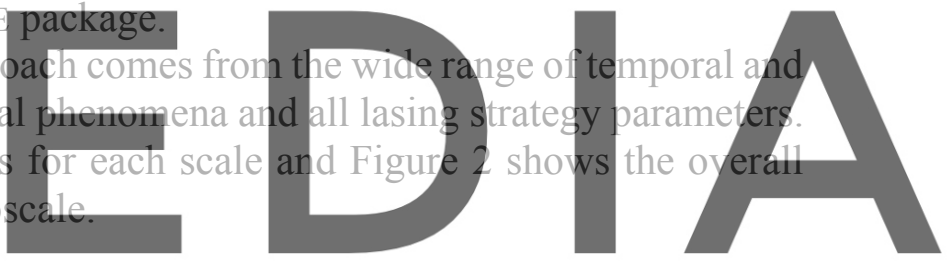

Register for free at https//www.Scipedia.com to download the version without the watermark

\begin{tabular}{lcccc} 
Level & Temporal domain & \multicolumn{3}{c}{ Spatial domain } \\
\hline 1 (Parts) & Days & $100 \mathrm{~mm}$ & $100 \mathrm{~mm}$ & $100 \mathrm{~mm}$ \\
\hline 2 (Layer) & Minutes & $100 \mathrm{~mm}$ & $100 \mathrm{~mm}$ & $1 \mathrm{~mm}$ \\
\hline 3 (Chessboard / Stripes) & Seconds & $1 \mathrm{~mm}$ & $1 \mathrm{~mm}$ & $0.1 \mathrm{~mm}$ \\
\hline 4 (Scan track) & 0.01 second & $1 \mathrm{~mm}$ & $0.1 \mathrm{~mm}$ & $10 \mu \mathrm{m}$ \\
\hline 5 (Melt pool) & 0.1 millisecond & $10 \mu \mathrm{m}$ & $10 \mu \mathrm{m}$ & $10 \mu \mathrm{m}$
\end{tabular}




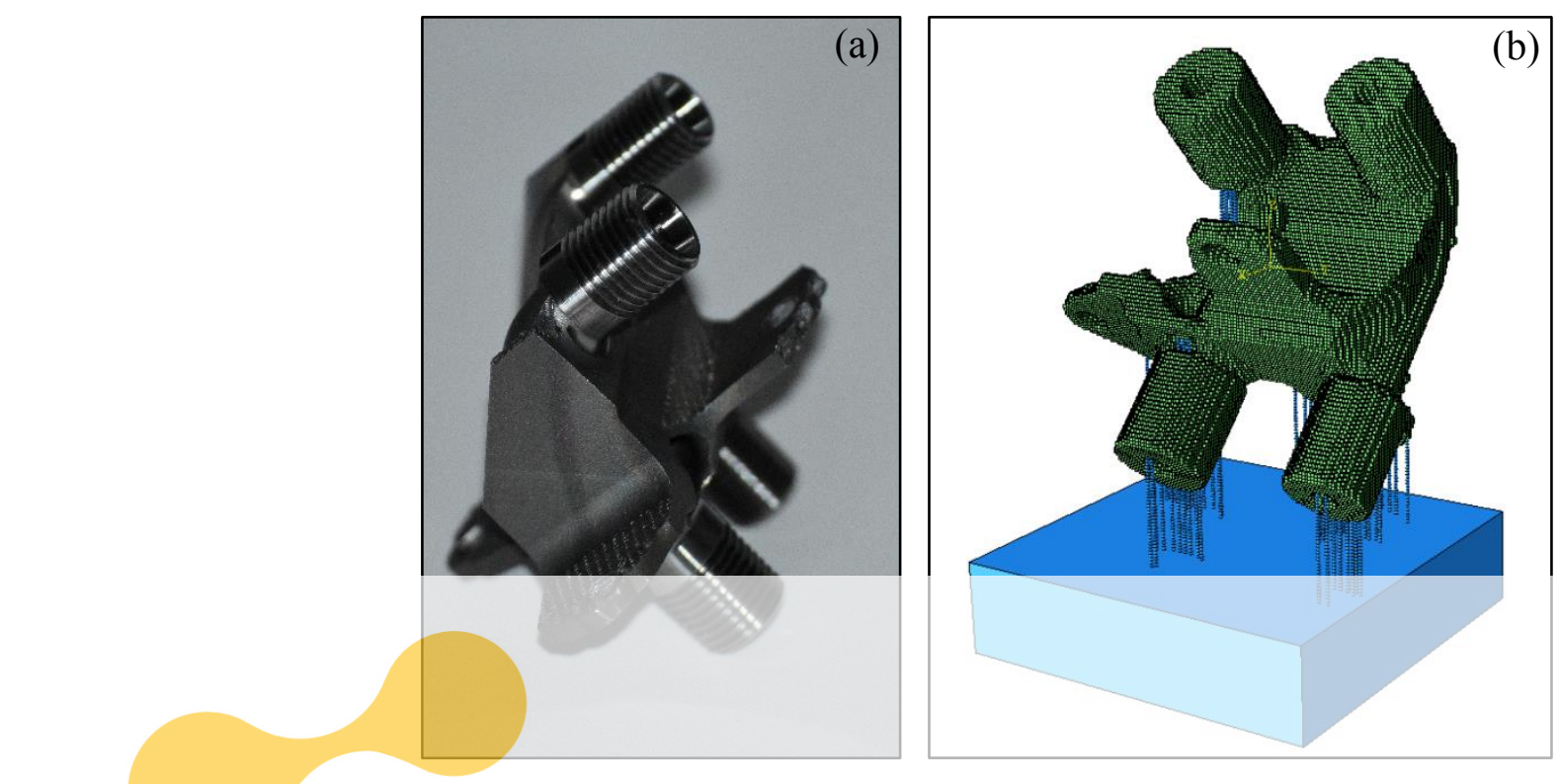

Figure 1: Original industrial hydraulic join (a) and hydraulic join ABAQUS model, supports and build plate (b)

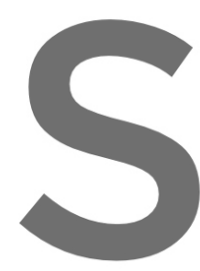

2.1 Level 1 description

The first level is the

manufactured. Its main

parts, identifying partial

layer, and evacuated thr
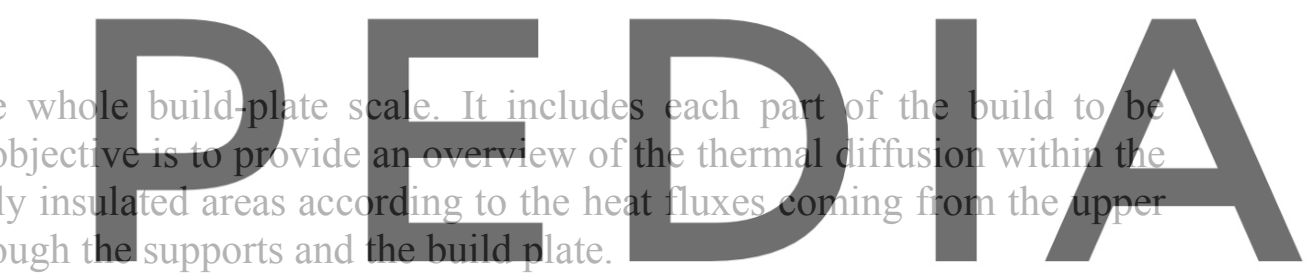

The parts are discretized with voxel elements at the height of the macro layers. Macrolayers

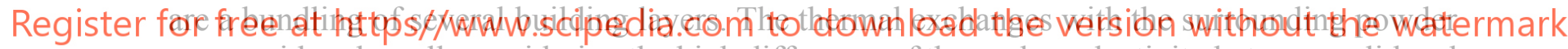
are considered small, considering the high difference of thermal conductivity between solid and powder materials. Hence, to avoid a fine mesh of the powder a small convection coefficient has been used on the lateral faces of the parts and the supports, as a first rough approximation.

The parts are attached to the build plate by the support structures. A prescribed temperature is set at the build plate's lower surface.

Primarily, the voxels are all deactivated, then consecutively activated and heated layer-wise using a volumetric heat source for a duration corresponding to the total duration of all layers within the macro layers.

The heat injected at the top of the activated regions is dissipated by the conduction within the underlying solid regions through thermal conduction transfer. Insulated regions (such as unsupported or overhang regions) suffer a temperature rise due to heat accumulation.

The computed thermal fields are then observed step by step. Regions of maximum or minimum temperatures can be registered and selected for further analysis at the second level.

\subsection{Level 1 results}

The part is $67 \mathrm{~mm}$ high, at a distance of $10 \mathrm{~mm}$ on a reduced build plate. This is a reduced 
model developed here as an example of the multiscale method. Level 1 geometries, comprising the part, the supports, and the build plate, are shown in Figure $1 \mathrm{~b}$.

For sake of simplicity, each step duration was calculated to scan throughout all the physical layers within each macro layer. A more precise scanning time could have been considered using real production duration.

The heat source considered delivered volumetric energy for each macro layer as represented in Figure 3a.

Isotropic aluminum temperature-independent material properties were considered as a first approximation. The USDFLD Abaqus subroutine was used to change the material properties during each activation, and to set the new macro layer temperature at the underlying body's temperature.

Level 1 results at macro layer $n^{\circ} 47$, which is the one where the highest temperature is reached during the build, are shown Figure $3 b$.

This upper macro layer, which reaches a maximum temperature of $720^{\circ} \mathrm{C}$, is thus identified and further investigated at Level 2.
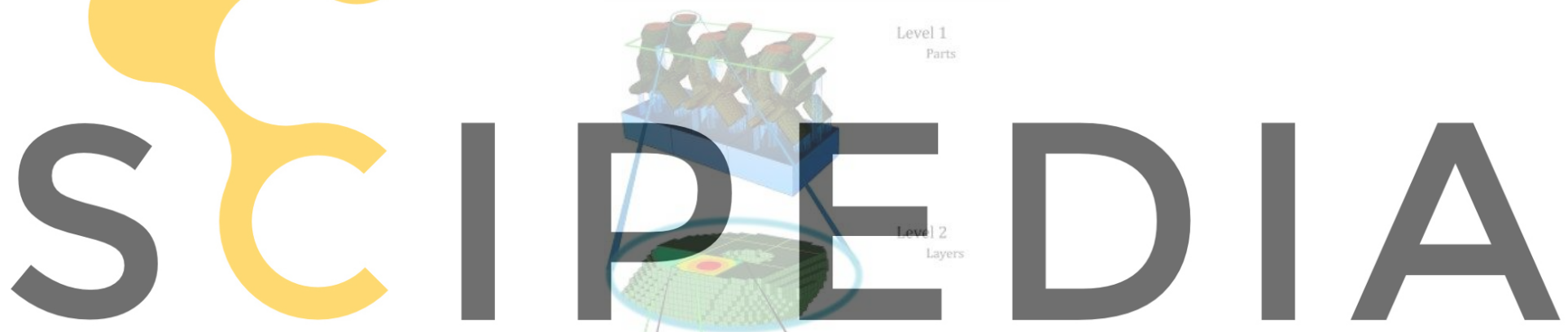

Register for free at https//www.scipedia.com to download the version without the watermark

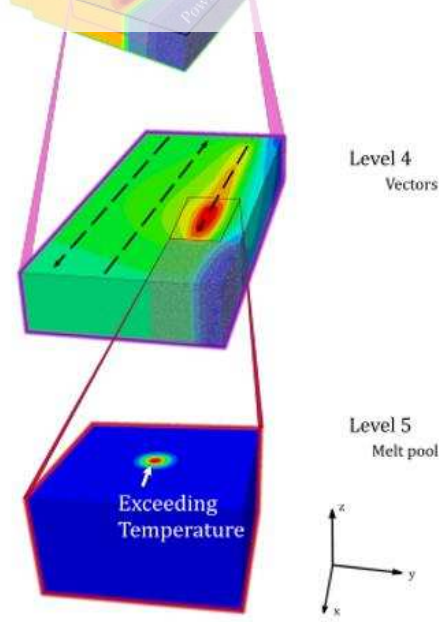

Figure 2: Overall multiscale approach 

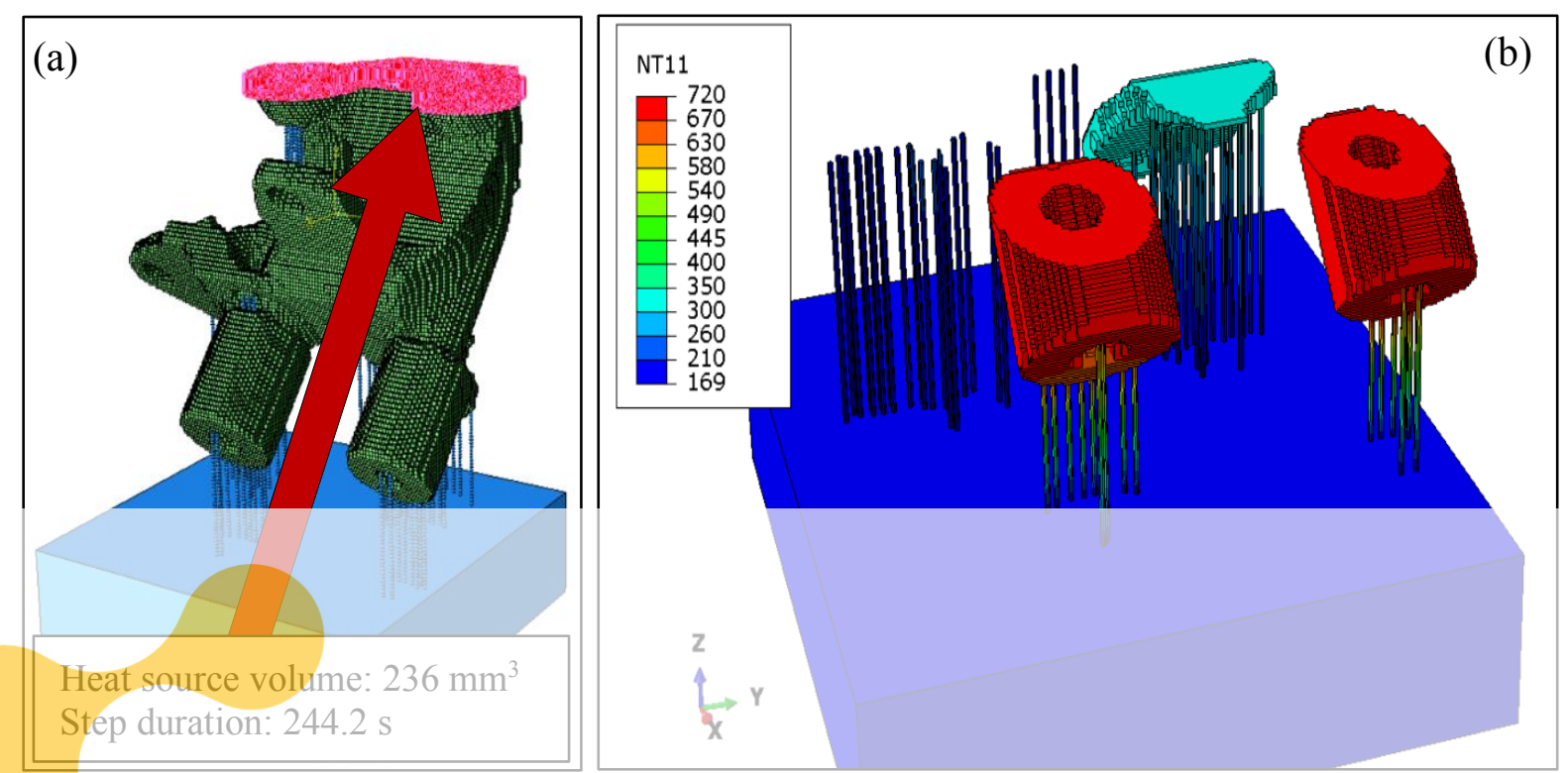

Figure 3: Level 1 model at (a) a random heated macrolayer, and (b) maximum temperature results at macrolayer
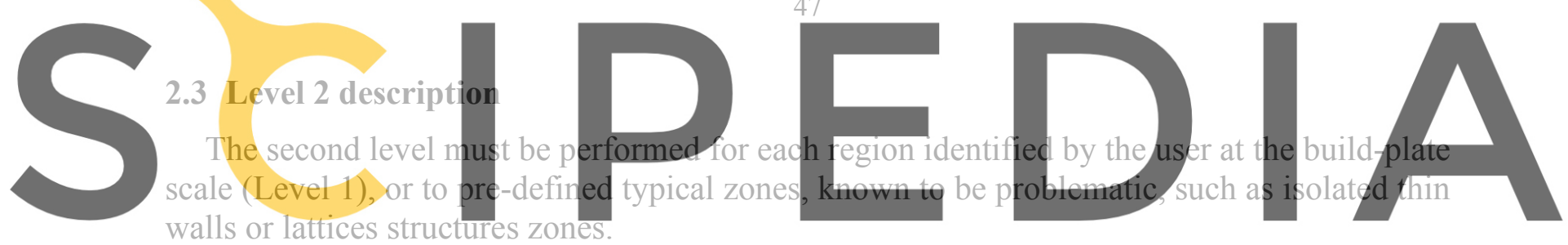

Level 2 is a zoom on specific layers identified at Level 1, or typical zones known to need

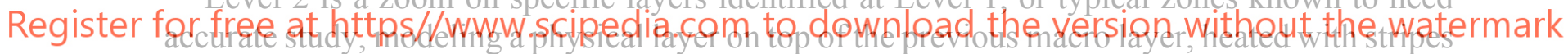
or islands patterns (according to the scanning strategy).

This level's objective is to observe the impact of the scanning strategy (island, stripes, etc.) by heating a particular sequence of islands on the layer.

The layer computed thermal history is looked upon to identify the island or the stripe where the maximum or minimum temperature is reached. Depending on the exact island locations and the scanning sequence, it is expected to reach different maximum temperatures for different sequence, and possibly to be able to optimize the heating sequences. This area's thermal fields are recorded and further investigated at Level 3 if necessary.

\subsection{Level 2 results}

Level 2 models the supports and the part up to the macro layer identified at Level 1 . The rest of the part and the build plate are deactivated as considered non useful to impact the local behavior.

A temperature boundary condition of $180^{\circ} \mathrm{C}$ (computed at Level 1) was set, here at the support base, since the Level 1 results showed that this temperature did not vary significantly during the macro layer heating. The optimal dimension of this sub model is also evaluated 
analytically and has been confirmed by several comparative simulations.

Surface heat flux loads were applied at the top surface of the macro layer (see Figure 4a). These loads' size and geometry correspond to a chessboard scanning strategy in this study case. All islands were heated sequentially, according to a typical chessboard sequence, and the zone with the maximum temperature (see Figure 4b) was chosen for deeper analysis at Level 3 modeling. Other scanning strategies, such as a stripe scanning strategy, could have been studied likewise, and even compared.

Figure 4 shows both the Level 2 model with an island-like loading, and the maximum temperature island result. This island will be zoomed in at Level 3.

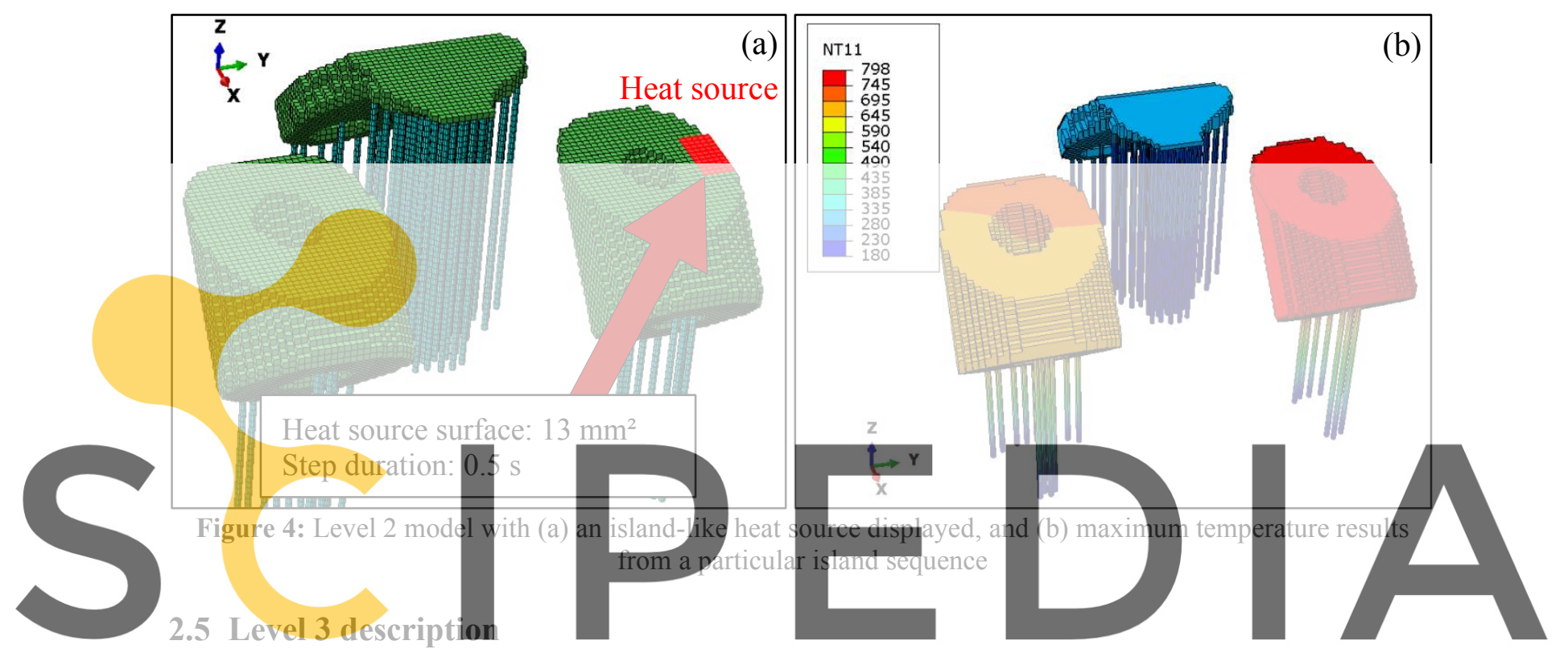

Level 3 is a zoom of the Level 2 identified region where a too high, or too low temperature

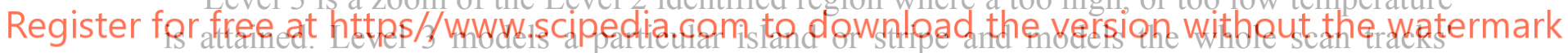

heating, as several lineic heat sources applied subsequently throughout this region.

The temperature fieids far from the island of interest, computed at Levei 2 , are used in Levei 3 as boundary conditions to reduce the geometrical domain.

With Level 3 computed thermal history, it is possible to identify the scan track which has suffered the maximum temperature and to compare various scan directions and the use of contouring strategies. This scan track zone will be further studied at Level 4, if needed.

\subsection{Level 3 results}

Level 3 model is a zoom on the zone identified at Level 2, and surface heat flux loads are applied subsequently on the upper surface. The shape of each load represents whole laser scan track lines.

Compared to Level 2, the model geometry was further reduced because at this time scale temperature variations are negligible outside this modelled zone (see Figure 5). The time increments and the meshes were both refined in comparison to the previous level.

Two sequences were tested: left to right and right to the left island-like loadings. Other sequences such as up to bottom and diagonals could also have been tested and compared in the 
same way.

Figure 5 shows the Level 3 model and maximum temperature scanning track results with island scan from right to left, chosen for further study at Level 4.
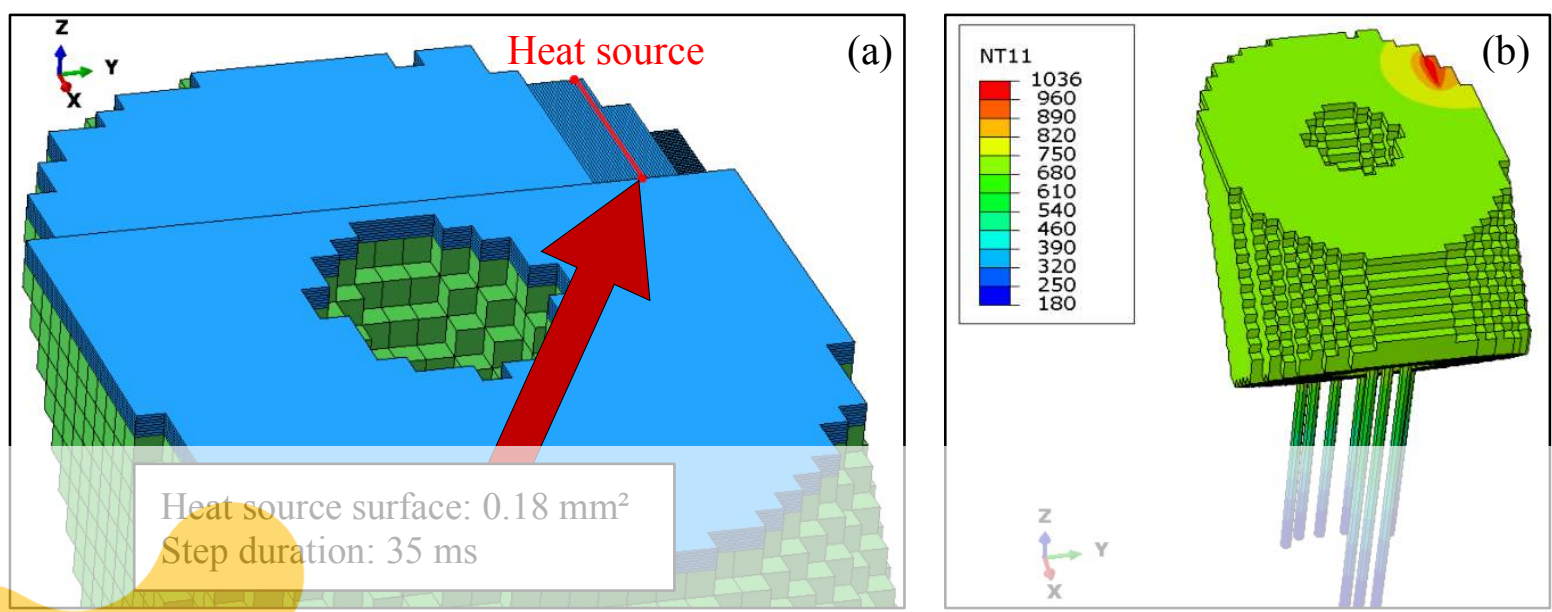

Figure 5: Level 3 model with (a) a particular scan track heat source, and (b) maximum temperature results from right to left

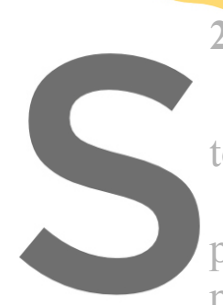

2.7 Level 4 description

Like the other levels,

too low temperature is

At this level, only sm

previous lineic heat sou

possible to identify whether the
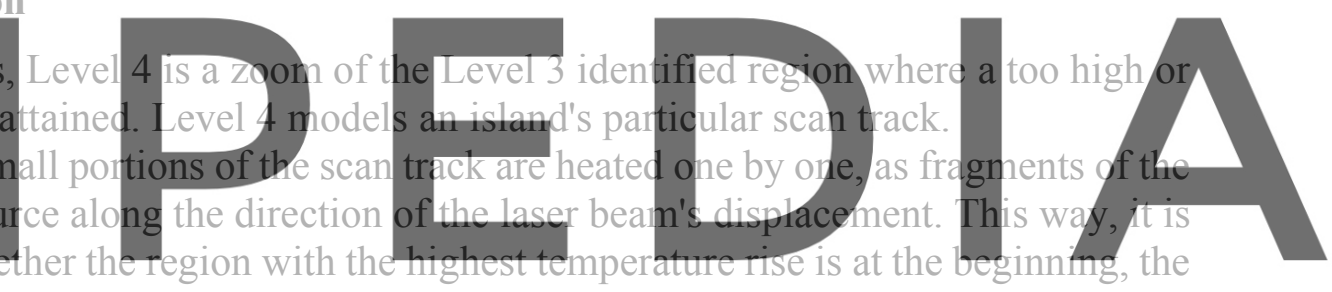

middle, or the end of the scan track. It is also possible to define the impact of a scanning

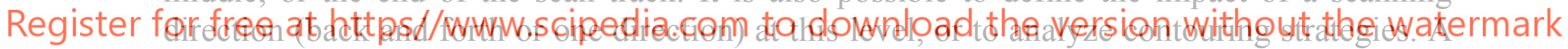

given scan track portion can be retained for further analysis at Level 5.

\subsection{Level 4 results}

At Level 4, the direction of the scan track is investigated. From Level 3, a particular scan track was identified, but at Level 4, a comparison regarding the scan track direction could be done.

Hence, compared to Level 3, the geometrical domain was again reduced, and both the meshes and time increments were refined.

At this level, small surface heat fluxes were applied upon the upper surface of the model (see Figure 6), these loadings simulate portions of the scan tracks at scanning speed. Averaging the laser beam's impact at this level allows to reduce the computational time (since the beam size is much smaller) but provides an overview of its impact on the track's different locations (at the beginning, the midway, or the end of it), as well as its direction.

Hence, at this level, the most suitable scanning direction can be found regarding the temperature's impact. We are also able to identify the portion in which the temperature would elevate the most. 
Both the model and the results of Level 4 are displayed in Figure 6. The particular track portion shown is the one with the higher temperatures and is considered in Level 5.
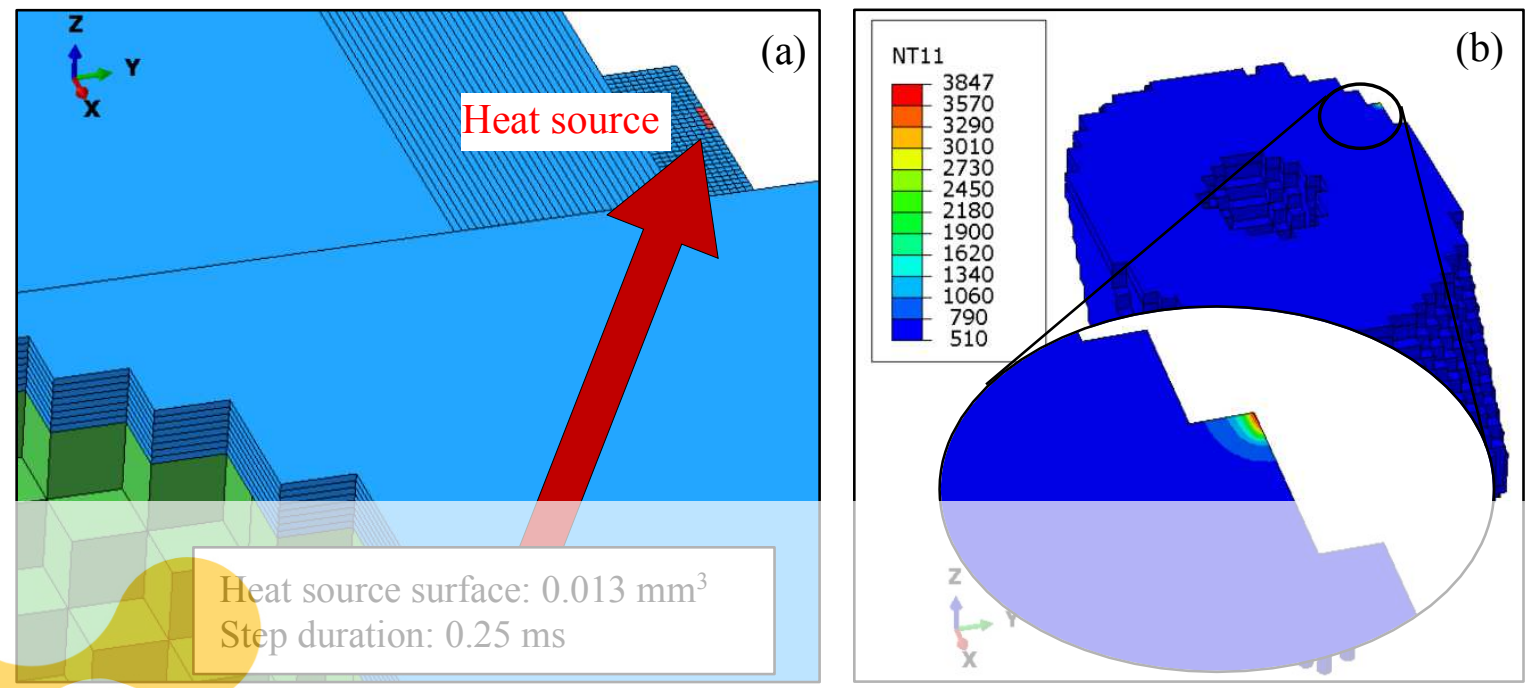

Figure 6: Level 4 model with (a) a particular scan track portion heat source and, (b) maximum temperature results from top to bottom direction

\subsection{Level 5 description}

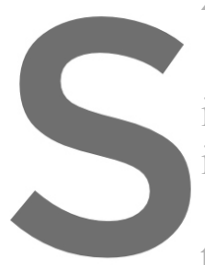

Level 5 is a zoom of

is applied as a surface he

included. We have not

At Level 5, it would

transfer within the liquid
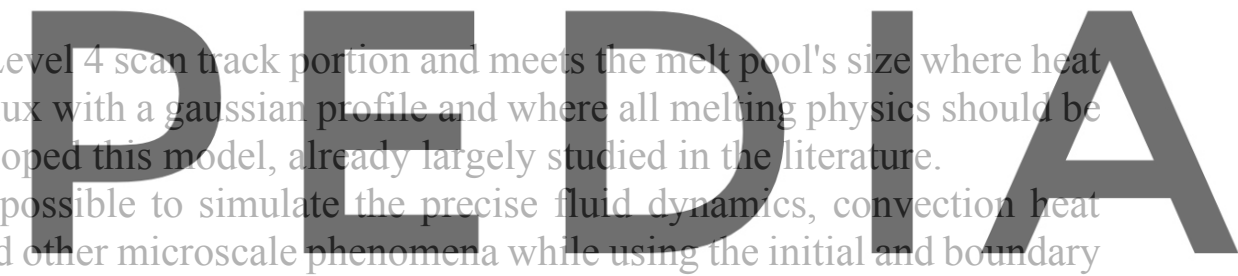

conditions computed at previous levels. In the scope of this study, Level 5 is performed using

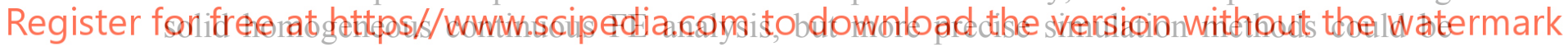
used.

\subsection{Level 5 results}

Level 5 is the last level of this multiscale approach. While it consists theoretically of a microscale model, it still considers the bodies as homogeneous continuum solids and the conduction in solids as the major heat transfer mechanism. Improvements would of course consist of modeling Level 5 with the thermal-fluid flow or thermal-fluid-vaporization models, which would be much more precise in capturing key physical factors at this scale [9], but these are outside the scope of the present work. Also, it is obvious that the part boundary should be refined at this level, but this do not change the spirit of our method.

A melt pool's size flux heat source is applied at the top of the upper layer at this level (see Figure 7). It is used to simulate the path of the laser beam as a punctual heat source application.

Compared to Level 4, the geometrical domain is reduced, and both the time steps and the meshes are refined.

Figure 7 shows both the Level 5 model, with a melt pool-like loading, and the results at the time of the maximum temperature. 
This Level 5 model could be used practically in adapting laser power at the beginning or the end particular scan tracks for instance, and could be used to study most phenomena considered in the literature such as the melt pool denudation, spatters, keyholes, etc.
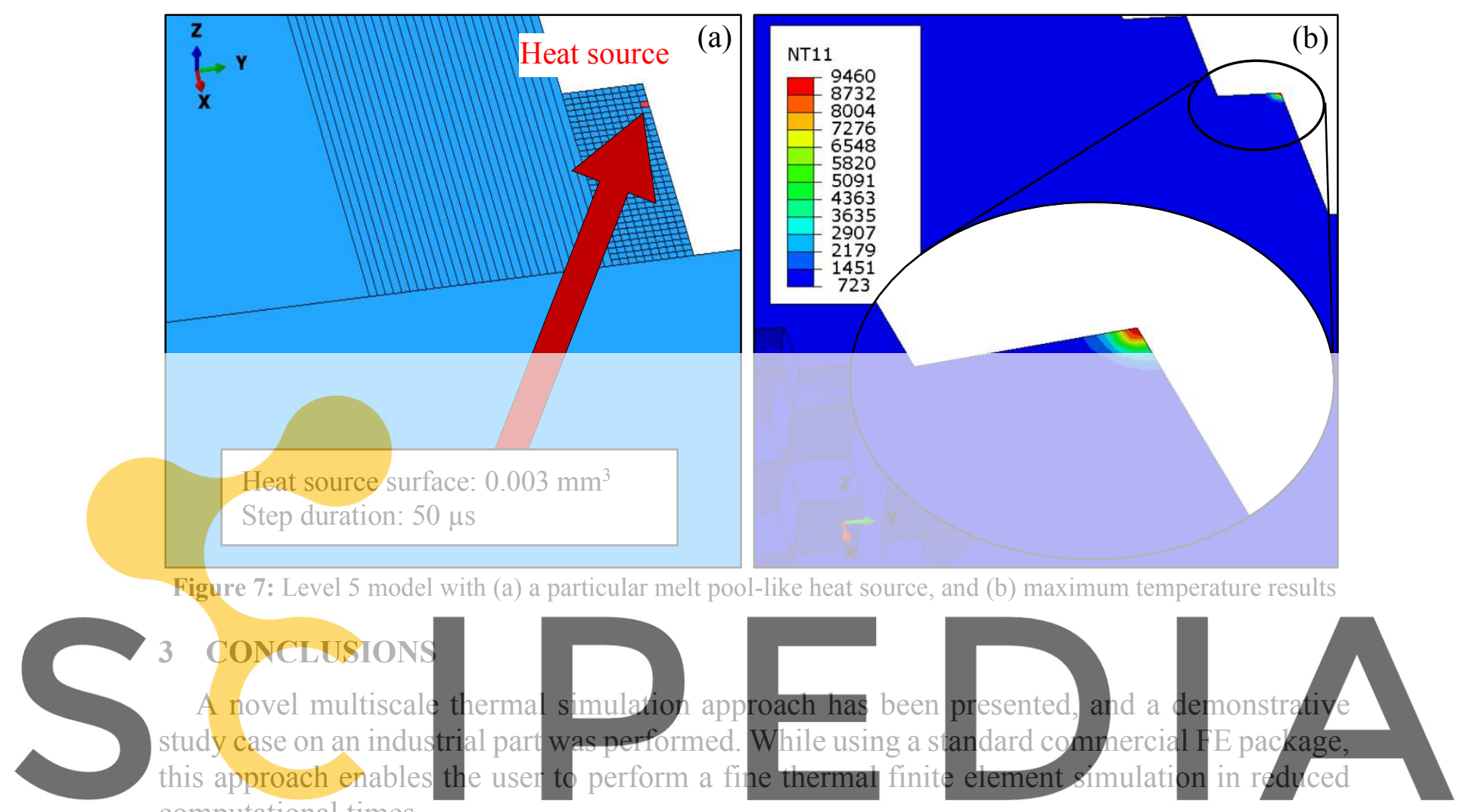

computational times.

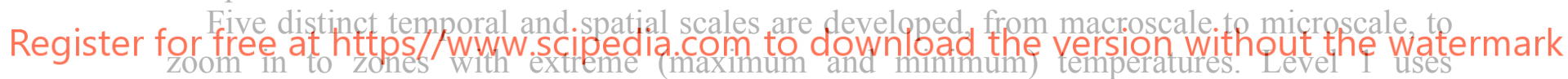

macrolayers and volumetric heat sources to model the whole build plate considering all the

parts and supports. Level 2 is zoom in to specific layers, where the scanning pattern (stripe or chessboard for instance) is studied. Level 3 uses lineic heat sources to model the whole scan tracks and determine the best scan track directions among the chosen configurations. Level 4 zooms in to scan track portions in order to determine the temperature fields at the beginning, the end and the middle of the selected scan tracks. Level 5 is similar to the melt pool models developed in the literature and could be used to study complex microscale phenomena.

The geometrical domains are gradually decreased with the zooms between the levels. The injected energy and the times used within each step correspond to those of the actual manufacturing process.

The zoom assumptions of the multiscale approach mainly rely on the assumption that the machine manufacturer's laser parameters yield parts with high densities (above 99\%), and regions with porosities are suffering a significant temperature rise.

The temperature fields computed at each level are used to identify regions for the next level and may be used to predict porosity location within the parts. A correlation between thermal results and porosity locations will be studied shortly. 
This model's precision and computational time will also be optimized using specific thermal assumptions.

\section{REFERENCES}

[1] P. Mercelis and J.-P. Kruth, «Residual stresses in selective laser sintering and selective laser melting», Rapid Prototyping Journal, vol. 12 (5), pp. 254-265, 2006.

[2] Z. Luo and Y. Zhao, «Numerical simulation of part-level temperature fields during selective laser melting of stainless steel 316L», International Journal of Advanced Manufacturing Technology, vol. 104, pp. 1615-1635, 2019.

[3] T.P. Moran, D.H. Warner and N. Phan, «Scan-by-scan part-scale thermal modelling for defect prediction in metal additive manufacturing», Additive Manufacturing, vol. 37, 2021.

[4] F. Dugast, P. Apostolou, A. Fernandez, W. Dong, Q. Chen, S. Strayer, R. Wicker and A. C. To, «Part-scale thermal process modeling for laser powder bed fusion with matrix-free method and GPU computing,» Additive Manufacturing, vol. 37, 2021.

[5] C. Li, C.H. Fu, Y.B. Guo and F.Z. Fang, «A multiscale modeling approach for fast prediction of part distortion in selective laser melting», Journal of Materials Processing Technology, vol. 229, pp. 703-712, 2016.

[6] N. Keller and V. Ploshikhin, «New method for fast predictions of residual stress and distortion of AM pa

[7] J. A. Mitchell, T. A.
porosity in additivel
[8] V. Benoist, L. Arna
including machinin
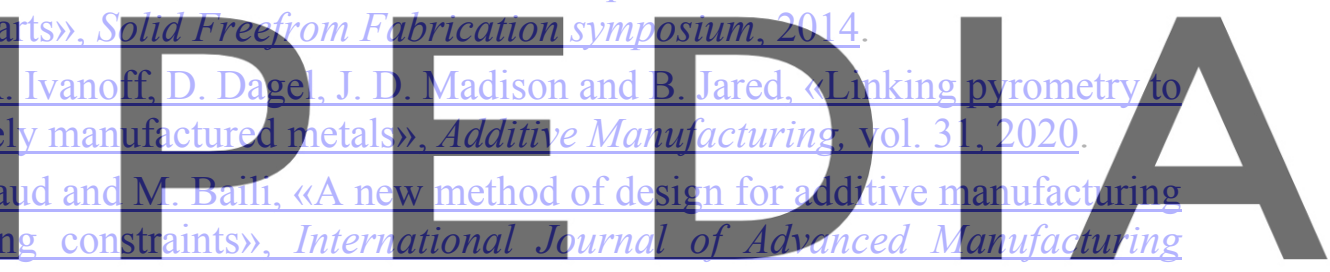

Technology, vol. 111, pp. 25-36, 2020.

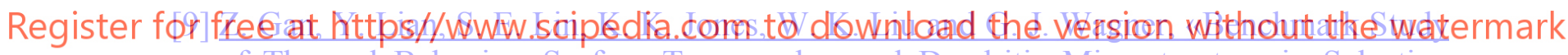
of Thermal Behavior, Surface Topography, and Dendritic Microstructure in Selective Laser Melting of Inconel 625», Integrating Materials and Manufacturing Innovation, vol. $\underline{8, p p .178-193,2019 .}$ 